\title{
$\begin{array}{ll}\text { Research Square } & \begin{array}{l}\text { Preprints are preliminary reports that have not undergone peer review. } \\ \text { They should not be considered conclusive, used to inform clinical practice, } \\ \text { or referenced by the media as validated information. }\end{array}\end{array}$
}

\section{Spatiotemporal expression of sonic hedgehog signalling molecules in the embryonic mesencephalic dopaminergic neurons of mice}

Manal T Hussein

Assiut University

Abdelraheim Attaai

Universite de Fribourg

Gamal Kamel

Assiut University

Doaa Mokhtar ( $\square$ doaamokhtar33@yahoo.com )

assuit university https://orcid.org/0000-0001-7975-3926

Research article

Keywords: Spatiotemporal expression, mesencephalon, Dopamine neuron, SHH, Boc, Gas1, MN9D

Posted Date: January 7th, 2021

DOI: https://doi.org/10.21203/rs.3.rs-36174/v2

License: (9) This work is licensed under a Creative Commons Attribution 4.0 International License.

Read Full License

Version of Record: A version of this preprint was published at Gene Expression Patterns on November 1st, 2021. See the published version at https://doi.org/10.1016/j.gep.2021.119217. 


\section{Abstract}

Background: Midbrain dopaminergic neurons (mDA) play an important role in controlling the voluntary motor movement, reward behaviour and emotion-based behaviour. Differentiation of mDA neurons from progenitors depends on several secreted proteins, such as sonic hedgehog $(\mathrm{SHH})$, and transcription factors. Different groups from mDA neurons arise from the varied patterns of SHH expression during development. The present study attempted to elucidate in mice embryo model, through in situ hybridization and immunohistochemistry 1 ) the possible role(s) of some $\mathrm{SHH}$ signalling components (Ptch1, Gli1, Gli2 and Gli3) in the spatiotemporal development of mDA neurons along the rostrocaudal axis of the midbrain and their possible roles in differentiation (E12, E14) and survival of mDA neurons (E18); 2) the main role of Boc (bioregional Cdon-binding protein), and Gas1 (growth arrest-specific1) as novel accessory receptors for the $\mathrm{SHH}$ in the development of mDA neurons 3 ) the significance of using the primary culture and/or the dopaminergic cell line (MN9D) for studying the development of mDA neurons.

Results: At E12 and E14, but not E18, only Ptch1 and Gli1 were expressed in ventrolateral midbrain domains. All examined SHH signalling molecules were not detected in mDA area. Whereas, in MN9D cells, many SHH signalling molecules were expressed and co-localized with the dopaminergic marker; tyrosine hydroxylase $(\mathrm{TH})$, and their expression were upregulated with $\mathrm{SHH}$ treatment of the MN9D cells.

Conclusion: These results suggest that mDA neurons differentiation and survival are independent of $\mathrm{SHH}$. Besides, MN9D cell line is not the ideal in vitro model for investigating the differentiation of mesencephalic dopaminergic neurons, and hence, the ventral midbrain primary culture might be favored over MN9D line.

\section{Background}

The midbrain dopaminergic mDA neurons are the most extensively studied neuronal subtypes because it is involved in several mental and neurodegenerative diseases. The mDA have a prominent function in movements, cognition, reward behaviour and emotions. The degeneration of the nigral dopaminergic neurons, substantia nigra pars compacta (SNpc, A9 group) and the subsequent loss of striatal target innervation underlie the motor symptoms of the Parkinson disease (PD), which is recognized as the second most common neurodegenerative disorder [1,2]. The best realization of molecular mechanisms that control the development of the mDA neurons will supply a valuable knowledge for the establishment of novel therapeutic alternatives and regenerative medical approaches for these diseases. Although the extensive studies performed on the development and function of the mDA system, the molecular mechanisms underlying, induction, specification, and survival of $\mathrm{mDA}$ neurons are still ambiguous.

Sonic Hedghog SHH is an early secreted morphogen by the floor plate and the notochord, which is associated with the patterning of the neural tube at the dorso-ventral axis and regulates numerous developmental processes [3]. The signalling pathways and the molecular determinants downstream of 
SHH have been characterized; the two cell surface receptors, the 12-transmembrane domain protein Patched (Ptch1) and the transmembrane G-coupled protein receptor Smoothened (Smo). After the binding of SHH to ptch1 receptor, the repression exerted by Ptch1 on Smoothened relived, allowing Smo to transduce SHH signalling. Afterwards, the glioma-associated oncogene (Gli) protein family members Gli1, Gli2 and Gli3 translocate to the nucleus and regulate the transcription of the SHH target genes [4-6]. Fate-mapping experiments clearly proved that all mDA neurons arise from progenitors in the midbrain floor plate that express SHH [7]. The expression pattern of SHH during mDA neurons development is considered a key player in providing different groups from mDA neurons $[8,9]$. In addition to Ptch1, several membrane-associated proteins are regarded to have a function as novel co-receptors that modulate SHH signalling activity. Gas1 (Growth arrest-specific 1), Cdo (cell-adhesion molecule-related/ downregulated by oncogenes), and Boc (bi-regional Cdon-binding protein) play overlapping and important roles during hedgehog signalling and mediate the ventral neural tube normal patterning [10]. Boc expression in the developing mDA neurons has not been yet demonstrated. The fused embryonic ventral mesencephalic and neuroblastoma cells (MN9D) is a dopaminergic cell line which commonly used as a model to demonstrate mDA neurons differentiation and maintenance. MN9D cells synthesize and express high levels of tyrosine hydroxylase, synthesize transport and release dopamine, express the neuron- specific markers such as neuronal nuclei (NeuN) and neurofilament proteins, express voltagesensitive sodium channels, express the vesicular monoamine transporter 2 (VMAT2) and aromatic amino acid decarboxylase (AADC) which are implicated in the dopamine storage and synthesis, respectively [11, 12]. MN9D cells express also the neuroectodermal stem cell marker (Nestin) and specific serotonergic markers such as the zinc-finger transcription factor (Gata2), PC12 ETS domain-containing transcription factor 1 (Pet1) [13].

The development of the mDA implies the sequential occurrence of developmental cascades of factors and a subsequent activation of transcription factors over time and space. The mDA neurons develop in a complex and hierarchical multi-step process divided roughly into 3 main stages. It starts at E7.5-9.5 with the regionalization of the midbrain and the establishment of the organizing centres such as the isthmus at the midbrain-hindbrain junction $(\mathrm{MHJ})$. The second step (E9.5-11.5) involves the specification of the progenitors to dopaminergic precursor cell fate, expressing various molecular markers. In the third stage (E 11.5-15.5), the dopaminergic precursors exit the cell cycle and begin to differentiate displaying early mDA markers. Finally, the mDA neurons become fully differentiated and functionally mature, expressing mature mDA properties [14-16].

Therefore, we aimed to investigate 1) possible role(s) of some SHH signalling components (Ptch1, Gli1, Gli2 and Gli3) in the spatiotemporal development of mDA neurons along the rostrocaudal axis of the midbrain; 2) the main role of Boc (bioregional Cdon-binding protein), and Gas1 (growth arrest-specific 1) as novel accessory receptors for the $\mathrm{SHH}$ in the development of mDA neurons 3 ) the significance of using the in vitro models, such as MN9D cell line and the ventral midbrain primary culture, as substitutions to decrease the number of utilized animals in studying the of the development of mDA neurons. 


\section{Results}

\section{Spatiotemporal characterization of SHH downstream components expression in the mDA neurons:}

We aimed to point out the roles of SHH signalling components in the development (differentiation and survival) of mDA neurons in vivo, so we characterized the temporal and spatial expression patterns of SHH receptor (Ptch1) and the transcription factors (Gli1, Gli2 and Gli3) at different embryonic ages E12, E14 (differentiation) and E18 (survival). To characterize the gene expression of the different SHH signalling components, we applied In situ hybridization (ISH), using Ptch1, Gli1, Gli2 and Gli3 mRNA probes on coronal and sagittal brain sections to detect their expression in $\mathrm{mDA}$ neurons at the transcriptional levels. Moreover, to analyze putative overlapping areas of expression between these molecules and $\mathrm{TH}$, consecutive sections have been processed for ISH and immunohistochemistry (IHC) for $\mathrm{TH}$.

As shown in Fig. 1A-C, at E12, Ptch1 expression was detected along the lateral parts of the developing midbrain, confirming previous observations, however, the exact location varies along the rostrocaudal axis. Rostrally, there are three main faint bands of expression along the lateral parts, with more prominent central one. The Ptch1 expression became stronger and restricted to the middle lateral domain of the mesencephalon (mes). Caudally, expression remained with more spreading dorsally. Gli1 expression (Fig. 1D-1F) was comparable to that of Ptch1, however, it was more diffuse dorsally and nearly absent caudally (arrow in Fig. 1D). Gli2 (Fig. 1G-1I) revealed higher levels of expression, compared to Ptch1 and Gli1 rostrally, diffuse in the dorsal half at the middle and caudal level. There was some overlap between Gli3 (Fig. 1J-1L) and Gli2 expression at their domain boundaries, however, its expression was lower rostrally, more diffuse and stronger in the dorsal half and the ventral fourth at the middle level whereas, caudally it was comparable to the patch 1 expression.

However, as illustrated in Fig. 2, Gli1 (Fig. 2A, 2D) and TH (Fig. 2C and 2F) expression domains appeared to be clearly separated all the way along the rostral-caudal axis. Similarly, we did not observe overlap between Ptch1 (Fig. 2B, 2E) and TH (Fig. 2C, 2F) expression domains.

To further elucidate the relation between the $\mathrm{SHH}$ signalling components and the differentiation of mDA neurons, we investigated the expression patterns of these components at E14 (Fig.3). We could not observe any obvious changes compared to E12. Using specific antisense ISH probes for Gli1, Gli2, Gli3 and Ptch 1 with mice midbrains at E14 (Fig.3A-3D) we observed that the expression of Ptch1 in the ventrolateral domain of the midbrains (arrow in $3 \mathrm{~A}$ ), Gli1 laterally and Gli2 and Gli3 were more confined to the dorsolateral wall of the aqueduct (arrow in Fig. 3C). Therefore, Ptch1 expression was complemented by Gli2 and Gli3 expression, whereas, neither of them was expressed in the mesencephalic $\mathrm{TH}$ immunoreactive domain of the consecutive sections. Moreover, we observed that Gli2 and Gli3 expression were the highest among the tested mRNA probes. In addition, Gli3 showed an expression in the red nucleus, a structure in the midbrain which originated from $\mathrm{SHH}$ expressing mesencephalic progenitors and involved in the motor coordination (asterisk in 3D). These results are specific, since by applying the corresponding sense probes no labeling was detectable (Fig. 3E-3H). The neuronal marker 
neurofilament (Fig. 3I) was used as a positive control for all ISH experiments. The developing cerebellum was used as a positive control for Gli2 (Fig. 3J) and Gli3 (Fig. 3K) probes. Furthermore, similarly to E12, along with the rostral-caudal axis, expression domains for Ptch1 (Figs 4A and 4B) and Gli3 (Figs 4C and 4D) showed no overlapping with the area of mesencephalic TH-expressing neurons (arrow in Figs $4 \mathrm{E}$ and $4 \mathrm{~F})$.

\section{Spatiotemporal expression patterns of Gas1 and Boc in the mesencephalon:}

To elucidate the possible role of Gas1 and Boc in the differentiation and/or survival of mDA neurons, we performed immunohistochemical analysis on coronal and sagittal brain cryosections from mouse E14 and E18 C57BL/ 6 embryos. Since Gas 1 and Boc are expressed in the developing mice cerebellum between E14.5 and E18 [17], therefore, we labeled cerebellar tissue with both antibodies to confirm the specificity of Gas 1 and Boc expression pattern in mDA area. We could not detect Boc nor Gas 1 expression in the area of the dopaminergic neurons. As shown in Fig. 5, at E14, the immunolabelling for both Boc (Fig. 5A) and Gas1 (Fig. 5C) was exclusively detected in the proliferative zone (arrowhead), but not in the mDA area where the TH positive neurons were existing (arrowhead in Fig. 5B and 5D). However, they were expressed in the rhombic lip (RL), ventricular zone (VZ) and choroid plexus (Chp) of the developing cerebellum as shown in Fig. 5E and 5F.

The expression of Gli3 (Fig. 6A), Ptch1 (6 Fig. B) and Gas1 (Fig. 6E) in mice midbrains at E18 were no longer observable in the midbrain, as determined by ISH and immunofluorescence. Moreover, consistent with the results obtained at E14, Gas1 was not detectable in mDA area (arrow in C and F) and the same investigation was observed with Boc. In the developing cerebellum (Fig. 6D and 6G), both Gli1 and Gas1 expression was found in the externally in the germinal layer (EGL), whereas choroid plexus (Chp) was additionally positive for Gas1. Collectively, these results suggest that $\mathrm{SHH}$ signalling components and $\mathrm{SHH}$ accessory receptors are not involved directly in the differentiation and survival of $\mathrm{mDA}$ neurons in vivo.

\section{In vitro studies:}

We decided to use MN9D cell line, a dopaminergic cell line model, which has been frequently used in many studies dealing with the differentiation of dopaminergic neurons [18]. We wanted to know whether the $\mathrm{SHH}$ signalling downstream components are expressed in this dopaminergic cell line. Treatment of MN9D cells with $1 \mathrm{nM} \mathrm{SHH}$ for 48 hours $(\mathrm{h})$ was done to enhance the expression of $\mathrm{SHH}$ and its downstream components in case they are not expressed. Subsequent expression of Gli1, Gli2, Ptch1 and SHH was examined by RT-PCR. Transcript expression for Ptch1 (Fig. 7A, 163bp), Gli1 (Fig. 7B, 492bp), Gli2 (Fig. 7C, 496bp), and SHH (Fig. 7D, 242bp) was detectable in control MN9D cells, and exogenous $\mathrm{SHH}$ treatment increased the intensity of Gli1, Gli2 and SHH bands. Indeed, quantification (Fig. 7E) revealed significant increase of Gli1 (1.55 \pm 0.11 fold), Gli2 (1.35 \pm 0.05 fold), and $S H H(1.53 \pm 0.14$ fold $)$ expression in $\mathrm{SHH}$-treated cells, compared to the controls ( ${ }^{\star} p<0.05$ and ${ }^{*} p<0.01$, using the unpaired Student's $t$-test, $\mathrm{n}=3)$. In contrast, Ptch1 expression did not change significantly between the control and treated cells ( $1.24 \pm 0.12$ fold, $n=3$ ). All results were normalized to the expression of Gapdh, a 
housekeeping gene. Interestingly, both non-differentiated cells (asterisk) and cells that have already acquired a neuronal morphology (arrow) were positive for Ptch1, thus matching the RT-PCR data. Same results were also obtained when MN9D cells were immune-labeled for Smoothened (Fig. 7F); both undifferentiated (asterisks) and differentiated (arrows) MN9D cells revealed immunoreactivity for Smoothened that was comparable in controls and SHH-treated cells. Finally, we examined whether THpositive MN9D cells also express Smoothened, and we observed a co-localization between TH and Smoothened markers, Fig. 7G. Notably, certain MN9D cells were immune-labeled with Smoothened but lack TH abundance. These results demonstrated that in contrast to the natural mouse mesencephalic tissue, MN9D cells express all SHH signalling components.

We further examined whether a mouse ventral midbrain primary cell culture form E14 ventral midbrain exhibited Smoothened expression, as the primary cell culture is considered more representative for in vivo status than the cell lines. We performed double immunostaining on these primary cultured cells from ventral midbrain of E14 mouse (kindly provided by Prof. Dr Bjoern Spittau) with antibodies against Smoothened receptors and $\beta$ III- tubulin (a marker for mature neurons) (Fig.7H). We observed that the primary cultured cells were expressing $\beta$ III- tubulin only, and unlike the MN9D cells, were negatively immunostained with the Smoothened marker.

\section{Discussion}

Although many studies have been conducted on the development and function of the mDA system, the molecular mechanisms underlying induction, specification and survival of mDA neurons are still growing. The present work aimed to investigate the expression, both temporal and spatial, of the SHH signalling components during the development of mice mDA neurons at E12, E14 and E18. SHH is an early secreted morphogen by the floor plate and the notochord, which is involved in the control of essential developmental processes, such as dorsoventral neural tube patterning, neural stem cell proliferation and cell fate specification [8]. The critical involvement of SHH in the differentiation of mDA neurons has been controversially debated in the literature.

Several previous studies had shown the critical role of SHH in the early development of mDA neurons. Cell fate mapping shows that mesencephalic cells expressing Gli1, present at the floor plate at E7.5-E9, are precursors to mDA neurons [19]. Fate-mapping experiments clearly established that midbrain dopaminergic neurons are derived from precursors in the floor plate that express SHH, Foxa1/2 and Lmx1a/b cells $[16,20,21]$. Hynes and colleagues observed that SHH can induce dopaminergic neurons specification in vitro, so they suggested that the $\mathrm{SHH}$ is sufficient and necessary for induction of mDA neurons [22]. Conversely, elimination of SHH signalling from the notochord by antibody blockade in vitro, or through gene targeting in mice (SHH mutant mice), prevents the differentiation of floor plate cells, the notochord degenerates, and motor neurons and most of ventral classes neurons of the neural tube are absent (dopaminergic and serotonergic neurons) [23, 24]. Using ISH with specific mRNA probes we showed that at E12 (Figs 1 and 2) and E14 (Figs. 3 and 4) expression of Gli family and Ptch1 revealed a mosaic pattern in the mesencephalic domains. At E12, the genes studied were predominantly confined to 
the medial lateral mesencephalic domains, while at E14 Gli2 and Gli3 were appeared dorsolaterally and Ptch1 and Gli1 expressed ventrolaterally. None of the studied genes was expressed in the same area of the mDA neurons (Figs 2C, 4E and 4F).

Blaess and his colleagues reported that SHH plays a key role in the induction of a dopaminergic phenotype by modulating the FoxA2 expression via Gli1 between E8 and E8.5 [25]. They added that in response to $\mathrm{SHH}$ signalling; Gli1 expression was upregulated, which is a Gli2 dependent, whereas Gli3 is suppressed by $\mathrm{SHH}$. The in vitro $\mathrm{SHH}$ treatment of E14 primary dissociated ventral mesencephalic cells significantly raised the number of surviving dopaminergic neurons [26]. These previous results declared that $\mathrm{SHH}$ is necessary for induction and survival of dopaminergic neurons. In contrast, more recent reports have indicated that $\mathrm{SHH}$ inhibits progenitors to obtain a dopaminergic cell fate $[27,28]$. In these studies, Wnt/ $\beta$-catenin signalling is considered as the major molecular determinant responsible for mDA neuron development. Using conditionally knock-out mice and/or gene inducible fate mapping other studies have concluded that $\mathrm{SHH}$ signalling is important for the mDA development during the early developmental stages E8.5- E11, and acting as an early morphogen of the floor plate and ventral midbrain [29].

Tang and his team performed fate-mapping studies using conditional mutant mice that lacked Smoothened receptor in the ventral neurogenic niche for dopaminergic neurons during different embryonic ages at E8.5 and E10.5 [30]. They observed that the removal of Smoothened at E8.5 lead to a significant loss in the DA at E10.5, while the removal of Smoothened at E10.5 lead to a transient loss at E12.5 and a sharp and continuous deficit was obvious in red nucleus, oculomotor and serotonergic neurons. Therefore, they suggested that $\mathrm{SHH}$ controls the development of the ventral midbrain neurons at the early embryonic stage.

Our results at E12 are in line with former observations of [8] and [30] at E11.5. A brilliant recent study analyzed the later embryonic stages and described different SHH signalling molecules [31]. In our study we have extended the analysis of dynamic spatiotemporal changes of different SHH signalling molecules investigating their expression along the rostrocaudal axis. We observed that the expression pattern at E12 (Fig. 3) persisted until E14 (Fig. 4). Interestingly, at E18, neither expression of Gli3 nor Ptch1 was detectable at any mesencephalic domain (Fig. 6). Consequently, according to our investigation and these studies we could conclude that $\mathrm{SHH}$ is indirectly functioning in the differentiation of mDA neurons.

Besides Patch1 and Cdon, both Boc, and Gas1 are identified as co-receptors that positively modulate SHH signalling activity. Gas1 and Boc play important and overlap ping roles during SHH patterning of the neural tube [10]. Gas1 and Boc are required and cooperate in the promotion of SHH signalling during embryonic development, as assessed by loss-of-function experiments [32]. Boc and Gas1 separately form discrete $\mathrm{SHH}$ receptor complexes with Ptch1 and are important for $\mathrm{SHH}$-dependent cell proliferation. Cdon and Boc promote $\mathrm{SHH}$-mediated cell fate specification and axon guidance. Among the function as tumor suppressor, Gas 1 is involved in the development of the CNS being initially expressed in ventral 
progenitors of the spinal cord and in a recent study, expression of Gas 1 has been detected in progenitors of the developing cortex and dentate gyrus [33].

The biological significance and requirement of Boc and Gas1 in SHH signalling has been highlighted in the work of [17], who reported that Gas1 and Boc interact with Ptch1 forming receptor complexes. Moreover, a SHH mutant protein capable to bind to Ptch1 but unable to bind Boc, Cdon or Gas1, hence, could not elicit SHH-dependent signalling and the cerebellar development was impaired in these mutants compared to control. Therefore, we concluded that there is absolute requirement for these co-receptors for the full activation of SHH signalling pathway. Based on these observations, we have studied distribution of Gas1 and Boc proteins in the mouse developing midbrain. At E14 Boc and Gas1 showed similar distribution, confined to the proliferative zone lining the aqueduct (Fig. 5), whereas, at E18 no expression could be detected for both (Fig. 6).

$M N 9 D$ is a cell line commonly used to study differentiation processes of mDA. Therefore, we have asked whether expression of the genes of interest in this line corresponds to our in vivo situation (Fig. 7). MN9D cells expressed Ptch1, and G/i family, and exogenous SHH treatment of these cells significantly increased the expression of Gli1, Gli2 and SHH. Since cultured MN9D cells maybe heterogeneous regarding their differentiation state, i.e. some cells represent progenitors while others have already acquired a neuronal phenotype, we assumed that the expression of the above-mentioned molecules would be restricted to the undifferentiated cells. However, our data have surprisingly shown that both Ptch1, and Smoothened proteins were also abundant in MN9D cells, independently of their differentiation state. Moreover, Smoothened was expressed in TH expressing MN9D cells. These results clearly demonstrate that expression of SHH signalling molecules in MN9D cells do not match that of native mouse mesencephalic tissue. We observed that the mouse ventral midbrain primary cell culture form E14 ventral midbrain was expressing BIII- tubulin (a marker for mature neurons), but not Smoothened. Therefore, the primary culture results, in part, comparable to our in vivo data and more representative for in vivo studies.

\section{Conclusions}

All SHH signalling components, Ptch1, Boc, Gas1and Gli family were absent in the mouse mDA area from E12-E18, suggesting that the differentiation and survival of mesencephalic dopaminergic neurons are $\mathrm{SHH}$-independent. Moreover, we provide evidence that, in contrast to the in vivo conditions, Gli1, Gli2, Ptch1 and Smoothened are expressed in the dopaminergic cell line, MN9D, implicating that this cell line might not be suitable for investigating the molecular pathways controlling the differentiation and maintenance of $\mathrm{mDA}$. The mouse ventral midbrain primary cell culture form E14 ventral midbrain might be favored over the MN9D cell line for in vitro studies for the differentiation and maintenance of $\mathrm{mDA}$. In the future, it would be interesting to elucidate whether other accessory receptors or alternative pathways for the SHH signalling exist and whether they contribute to the differentiation and survival of mDA neurons.

\section{Methods}




\section{Animals}

The used pregnant mice (C57B L/6J) were purchased from The Jackson Lab. This work was performed according to the German Ethical Guidelines for Laboratory Animals recommendations. The protocol was sanctioned by the ethics committee of the City of Freiburg, (authorizations: G11/56 and X-16/10F), and the Institutional Animal Care and Use Committee of the Freiburg University.

Different embryonic ages of wild-type mice (C57B L/6J) have been used, starting from E12, E14 and E18, to elucidate the role of SHH accessory receptors (Boc and Gas1) and SHH downstream components (Ptch1 receptors, Gli1, Gli2 and Gli3 transcription factors) in the development (differentiation and survival) of the mDA neurons. Pregnant C57BL/6J mice were sacrificed by cervical dislocation. Embryos were obtained by the cesarean section, rinsed with $1 \mathrm{xPBS}(\mathrm{pH} 7.4)$, fixed overnight at $4^{\circ} \mathrm{C}$ in $0.1 \mathrm{M}$ PBS containing 4\%PFA. After fixation, embryos were rinsed 3x PBS for 30 minutes (min). and then cryoprotected in $15 \%$ sucrose in PBS overnight at $4^{\circ} \mathrm{C}$. Later, embryos were immersed in $30 \%$ sucrose in PBS and kept at $4{ }^{\circ} \mathrm{C}$ overnight until the embryos sank. Following overnight immersion in sucrose embryos were coated with Tissue Tek (OCT compound), frozen in liquid nitrogen and finally stored at $20^{\circ} \mathrm{C}$ until further processing. Sagittal and coronal serial sections at $10 \mu \mathrm{m}$ thickness were sectioned by Cryostat, mounted on super-frost slides, and air dried for 40 min before performing in-situ hybridization or immunohistochemical staining.

\section{Antibodies}

Mouse anti-TH (MAB318, Millipore) and rabbit anti-TH (AB152) (Merck-Millipore, Darmstadt, Germany) were used for immunoperoxidase light microscopy. Goat anti-Gas1 (AF2644, R\&D Systems) and anti-Boc (AF2385, R\&D Systems) used for immunofluorescent (IF) staining. Goat anti-Ptch1 (G-19; sc-6149, Santa Cruz Biotechnologies, Heidelberg, Germany), rabbit anti-Smoothened (ab38686, Abcam, Cambridge, UK) for IF staining to MN9D cell line. Secondary antibodies were goat anti-mouse or goat anti-rabbit IgG coupled to horseradish peroxidase, donkey anti-mouse, donkey anti-goat or donkey anti-rabbit IgG Alexa 568 (Dianova, Germany). DAPI- Fluoromount G (Biozol, Germany) was used for counterstaining of nuclei and Aquatex (Merck-Millipore, Germany) for mounting.

\section{Immunohistochemistry (IHC)}

\section{$D A B$ staining}

Frozen sections were dried at room temperature (RT) for 30 min then washed once for 10 min in 1x PBS. Antigen retrieval was performed in $0.1 \mathrm{M}$ citrate buffer solution $(\mathrm{pH} 6)$ for $3 \mathrm{~min}$ using a microwave at (600 Watt). Sections were washed 3x 5 min each with 1x PBS, incubated in 30\% hydrogen peroxide $\left(\mathrm{H}_{2} \mathrm{O}_{2}\right)$ in methanol $\left(75 \mathrm{ml}\right.$ methanol $\left.+750 \mu \mathrm{H}_{2} \mathrm{O}_{2}\right)$ for 30 min to block the endogenous peroxidase activity. Afterwards, the cryosections were washed for 5 min with 1x PBS, incubated with blocking solution [10\% normal donkey serum (NDS) $+0.2 \%$ Triton-X100/PBS] for $2 \mathrm{~h}$ at RT, incubated with rabbit anti-TH diluted at 1:500 in blocking solution overnight at $4^{\circ} \mathrm{C}$. On the second day sections were rinsed in PBS $3 \times 5$ min each, 
incubated with secondary antibody conjugated to biotin (diluted in $1.5 \%$ NDS + 0.2\% Triton-X100/PBS) for $2 \mathrm{~h}$ at RT, followed by incubation with avidin and biotinylated horseradish-peroxidase macromolecular complex ( $A B C$ kit) for 45 min. Finally, the antigen-antibody reaction was visualized using the $D A B$ peroxidase substrate (15-20 min), rinsed with distilled water, mounted with Aqua-Tex and analyzed with a bright field (Axioplan2, Zeiss) microscope.

\section{Immunofluorescent staining}

The sections were treated as mentioned above without $\mathrm{H}_{2} \mathrm{O}_{2}$ treatment. The cerebellum was used as a positive control for Gas and Boc. They were incubated with the primary antibodies (anti-Gas 1 or anti-Boc at dilutions 1:200 and 1:100, respectively) overnight at $4^{\circ} \mathrm{C}$. On the second day, the sections were washed in PBT ( $3 \times 10$ min each), incubated with Alex Fluor ${ }^{\circledR}$ secondary antibodies for $2 \mathrm{~h}$ at $\mathrm{RT}$ in a dark place. Finally, the sections were washed $3 \times 5$ min each in 1xPBS, mounted with Flouromount-G mixed with 4'-6diamidino-2-phenylindole (DAPI) diluted 1:1000 in PBS for counterstaining the nuclei. Then the reaction was visualized and imaged under the fluorescence microscope (Axioplan2, Zeiss).

\section{In situ hybridization (ISH)}

This technique was performed, as described by [34], to detect specific ribonucleic acid sequence using labelled mRNA probe with a molecular marker, such as Digoxigenin (DIG; non-radioactive antibody) to investigate the expression of a gene of interest at the transcriptional level. ISH was performed on frozen sections using DIG labelled mRNA probes (Roche, Mannheim, Germany) for the SHH signalling components such as, Ptch1 receptor, Gli1, Gli2 and Gli3 transcriptional factors, neurofilaments (NF) and $\mathrm{TH}$. The sense probes were used as negative controls. The neurofilament marker was used as a positive control for all ISH experiments. In addition, cerebellum was used as a positive control for Gli1, 2, 3. ISH was performed in RNase free conditions and all used solutions were prepared using DEPC water and under sterile conditions. The DIG-labelled RNA probes were diluted with Tris-EDTA buffer (1:10), afterwards, the diluted DIG labelled RNA probes were added to $150 \mu$ l of hybridization buffer and applied on the freshly dried frozen sections. The buffer box containing the sections were placed in humidified chamber and incubated overnight at $68^{\circ} \mathrm{C}$. On the second day, slides were washed in washing buffer at $70^{\circ} \mathrm{C}$ for $10 \mathrm{~min}$. Post hybridization washes were performed twice for $30 \mathrm{~min}$ at $70^{\circ} \mathrm{C}$ in washing buffer, immersed in 1x MABT (maleic acid buffer with tween) at RT for 30 min twice. Afterwards, the sections were blocked with blocking reagent ( $1.2 \mathrm{ml}$ lamb serum: $4.8 \mathrm{ml} \mathrm{MABT})$ for $2 \mathrm{~h}$, followed by incubation with sheep anti-DIG-AP (anti- DIG conjugated to alkaline phosphatase (AP) in blocking reagent (1:1500) at RT overnight in a humid chamber. On the third day, sections were washed with 1x MABT for 20 min at RT, covered with $400 \mu \mathrm{l}$ MABT twice for $20 \mathrm{~min}$ /each, covered with $400 \mu$ l three times with filtered AP buffer for $10 \mathrm{~min}$. To visualize the DIG- labelled mRNA probes; Nitro-blue tetrazolium chloride in combination with 5-bromo-4-chloro-3'-indolyphosphate p-toluidine (NBT/BCIP, Roche) was used as a substrate (colouring solution) overnight at RT. On the fourth day, the sections were washed in $1 x$ PBS, mounted with aquatex and examined with a bright field (Axioplan2, Zeiss) microscope. 


\section{In vitro cell/tissue culture}

MN9D is a dopaminergic cell line, which was established by hybridoma fusion of cells from E14 murine mesencephalic cells with neuroblastoma cell line N18TG2 [35]. We aimed to investigate whether the SHH signalling downstream components are expressed in this dopaminergic cell line. Cells were plated on

poly-D-lysine (PDL) coated 12-mm² glass coverslips in 24-well plates at a density of $5 \times 10^{4}$ cells/ coverslips. Cells were cultured in Dulbecco's Modified Eagle Medium/F12 (DMEM/F12 1:1), supplemented with $5 \%$ fetal bovine serum (FBS) and 1\% penicillin/streptomycin/ neomycin (to prevent bacterial contamination) and maintained at $37^{\circ} \mathrm{C}, 5 \% \mathrm{CO}_{2}$, and $95 \%$ humidified air atmosphere. The cells were passaged when they reached approximately $80-90 \%$ confluency. Cells were treated with $1 \mathrm{nM} \mathrm{SHH}$ for 48h. Subsequently, after 2 days in-vitro (DIV2) controls and SHH treated cells were either fixed for IF or processed for RNA extraction and reverse transcription (RT-PCR). Coverslips of foetal mouse mesencephalic neural progenitor cells (NPCs) were kindly provided by Prof. Bjoern Spittau.

\section{Immunocytochemistry}

The coverslips of the SHH-treated MN9D cells and the vehicle control were fixed with 4\% PFA for 20 min at RT and washed $3 \times 5$ min in PBS. Afterwards, $1 \%$ SDS was used to permeabilize the cell membrane, then blocked in $1 \%$ bovine serum albumin (BSA) in PBS for 30 min at RT. MN9D cells were incubated with primary antibodies; rabbit anti-Smo (1:100; Abcam, ab38686), goat anti-Patch1 (1:100; Santa Cruz Biotechnology, SC6149), mouse anti-TH (1:1000; Merck-Millipore, Darmstadt, Germany, MAB318) diluted in PBS overnight at $4^{\circ} \mathrm{C}$. The primary cultured mesencephalic dopaminergic neurons were incubated with primary antibodies; mouse anti BIII-tubulin-c (1:100; Developmental Studies Hybridoma Bank; DSHB Hybridoma Product E7) and rabbit anti-Smo (1:100; Abcam, ab38686) diluted in PBS overnight at $4^{\circ} \mathrm{C}$. On the following day, cover slips were washed with PBS $3 \times 5$ min each, incubated with the corresponding secondary antibodies coupled to Alexa Fluor 594 or Alexa Fluor 488 for $2 \mathrm{~h}$ at RT. The coverslips were washed in PBS $3 \times 5$ min, mounted with mixture of Flouromount-G and DAPI for counterstaining of the nuclei.

\section{RT-PCR (reverse transcriptase-polymerase chain reaction)}

Total RNA was isolated from SHH-treated MN9D cells and control, reverse transcribed for Synthesis of CDNAand processed for PCR, as described earlier [11].

\section{PCR (Polymerase chain reaction)}

The primers used for Gli1, Gli2, SHH, and ptch1 were supplied from the Gene-bank. Conventional PCR was performed on an Eppendorf Mastercycler (Wesseling, Germany). For detection of the active transcribed genes from the cDNAs, the following protocol was used; denaturation of the DNA for $5 \mathrm{~min}$ at $95^{\circ} \mathrm{C}$, the optimum number of cycles -depending on primer pairs- of PCR amplification were performed at following conditions: denaturation for $30 \mathrm{sec}$ at $95^{\circ} \mathrm{C}$, primer annealing for $45 \mathrm{sec}$, and elongation for $45 \mathrm{sec}$ at $72^{\circ} \mathrm{C}$. Final extension for 10 min at $72^{\circ} \mathrm{C}$ was terminated by rapid cooling at $4^{\circ} \mathrm{C}$. PCR products were run 
in agarose gel electrophoresis, and the bands of the reaction products were visualized using a UV transilluminator and TS view software.

To elucidate whether the genes of interest were up- or down-regulated, the reciprocal of each calculated value was estimated. The size and intensity of the bands of interest was measured using the ImageJ software. The optical density of each amplified band was calculated and numerically expressed as the relative density in comparison to the optical density of the background. The ratio of the determined intensity of the bands of interest was divided by the ratio of the intensity of the bands from "housekeeping" gene, GAPDH. The control was set to 1 and the different conditions were normalized to the control.

\section{Statistical analysis}

Data were presented as the mean \pm SEM and were statistically analyzed using unpaired Student's t-test, to compare treated group with the untreated control. Differences were considered statistically significant at $\left(* \star * P \leq 0.001,{ }^{\star *} P \leq 0.01\right.$ and $\left.* P \leq 0.05\right)$. $p>0.05$ was considered not statistically significant. GraphPad Prism 6 (GraphPad Software Inc.) was the used software for statistical analysis.

\section{List Of Abbreviations}

$\begin{array}{ll}\text { Boc } & \text { brother of CDO } \\ \text { CNS } & \text { central nervous system } \\ \text { DAB } & \text { Di-Amino-benzodiazepine } \\ \text { E } & \text { Embryonic day } \\ \text { Gas1 } & \text { growth arrest-specific protein 1 } \\ \text { Gli1 } & \text { Gli protein 1 } \\ \text { Gli2 } & \text { Gli protein 2 } \\ \text { Gli3 } & \text { Gli protein 3 } \\ \text { IHC } & \text { immunohistochemistry } \\ \text { ISH } & \text { In situ hybridization } \\ \text { mDA } & \text { midbrain dopaminergic neurons } \\ \text { MHJ } & \text { midbrain-hindbrain junction } \\ \text { PBS } & \text { phosphate buffer saline }\end{array}$


PFA

Paraformaldehyde

Ptch1

Patched- receptor 1

RT- PCR

Reverse transcription-polymerase chain reaction

RT

Room temperature

$\mathrm{SHH}$

sonic hedgehog

Smo

Smoothened receptor

SNpc, A9 group

substantia nigra pars compacta

$\mathrm{TH}$

Tyrosine hydroxylase

VMAT2

Vesicular monoamine transferase 2

\section{Declarations}

Acknowledgements

This work was supported by a scholarship from the Egyptian Ministry of Higher Education for both Manal Hussein and Abdelraheim Attaai, and by Deutsche Forschungsgemeinschaft (Kr1477/10-3).

\section{Authors' contributions}

GK designed experiments, coordinated, and supervised the study.

$\mathrm{MH}, \mathrm{AA}, \mathrm{DM}$ designed and carried out experiments, performed data analysis and wrote the manuscript, which all authors read and approved.

\section{Funding}

The current study has not any fund from any organizations or institutions.

\section{Ethics approval and consent to participate:}

This study was carried out in strict accordance with the National Health and ethical regulations. Care of animals was in accordance with institutional guidelines. The Committee on the Ethics of Animal Experiments of the University of Freiburg approved the protocol of this work (Permit Number: X-07/29A).

\section{Consent for publication}

Not applicable. 
Availability of data and materials

The datasets used and/or analysed during the current study are available from the corresponding author on reasonable request.

Competing interests

The authors declare that they have no competing interests

\section{References}

1. Bjorklund A, Dunnett SB: Dopamine neuron systems in the brain: an update. Trends in neurosciences 2007, 30(5):194-202.

2. Morales $M$, Margolis EB: Ventral tegmental area: cellular heterogeneity, connectivity and behaviour. Nature reviews Neuroscience 2017, 18(2):73-85.

3. McMahon AP, Ingham PW, Tabin CJ: Developmental roles and clinical significance of hedgehog signalling. Current topics in developmental biology 2003, 53:1-114.

4. Bayly RD, Brown CY, Agarwala S: A novel role for FOXA2 and SHH in organizing midbrain signalling centers. Developmental biology 2012, 369(1):32-42.

5. Chen Y, Struhl G: Dual roles for patched in sequestering and transducing Hedgehog. Cell 1996, 87(3):553-563.

6. Ingham PW, McMahon AP: Hedgehog signalling in animal development: paradigms and principles. Genes \& development 2001, 15(23):3059-3087.

7. Yan $\mathrm{CH}$, Levesque $\mathrm{M}$, Claxton S, Johnson RL, Ang SL: Lmx1a and Imx1b function cooperatively to regulate proliferation, specification, and differentiation of midbrain dopaminergic progenitors. The Journal of neuroscience : the official journal of the Society for Neuroscience 2011, 31(35):1241312425.

8. Blaess S, Bodea GO, Kabanova A, Chanet S, Mugniery E, Derouiche A, Stephen D, Joyner AL: Temporal-spatial changes in Sonic Hedgehog expression and signalling reveal different potentials of ventral mesencephalic progenitors to populate distinct ventral midbrain nuclei. Neural development 2011, 6:29.

9. Joksimovic M, Anderegg A, Roy A, Campochiaro L, Yun B, Kittappa R, McKay R, Awatramani R: Spatiotemporally separable Shh domains in the midbrain define distinct dopaminergic progenitor pools. Proceedings of the National Academy of Sciences of the United States of America 2009, 106(45):19185-19190.

10. Allen BL, Song JY, Izzi L, Althaus IW, Kang JS, Charron F, Krauss RS, McMahon AP: Overlapping roles and collective requirement for the coreceptors GAS1, CDO, and BOC in SHH pathway function. Developmental cell 2011, 20(6):775-787. 
11. Hermanson E, Joseph B, Castro D, Lindqvist E, Aarnisalo P, Wallen A, Benoit G, Hengerer B, Olson L, Perlmann T: Nurr1 regulates dopamine synthesis and storage in MN9D dopamine cells. Experimental cell research 2003, 288(2):324-334.

12. Kim KS, Kim CH, Hwang DY, Seo H, Chung S, Hong SJ, Lim JK, Anderson T, Isacson O: Orphan nuclear receptor Nurr1 directly transactivates the promoter activity of the tyrosine hydroxylase gene in a cell-specific manner. Journal of neurochemistry 2003, 85(3):622-634.

13. Osterberg N, Wiehle M, Oehlke O, Heidrich S, Xu C, Fan CM, Krieglstein K, Roussa E: Sim1 is a novel regulator in the differentiation of mouse dorsal raphe serotonergic neurons. PloS one 2011, 6(4):e19239.

14. Abeliovich A, Hammond R: Midbrain dopamine neuron differentiation: factors and fates. Developmental biology 2007, 304(2):447-454.

15. Andersson E, Tryggvason U, Deng Q, Friling S, Alekseenko Z, Robert B, Perlmann T, Ericson J: Identification of intrinsic determinants of midbrain dopamine neurons. Cell 2006, 124(2):393-405.

16. Ono Y, Nakatani T, Sakamoto Y, Mizuhara E, Minaki Y, Kumai M, Hamaguchi A, Nishimura M, Inoue Y, Hayashi $\mathrm{H}$ et al: Differences in neurogenic potential in floor plate cells along an anteroposterior location: midbrain dopaminergic neurons originate from mesencephalic floor plate cells. Development 2007, 134(17):3213-3225.

17. Izzi L, Levesque M, Morin S, Laniel D, Wilkes BC, Mille F, Krauss RS, McMahon AP, Allen BL, Charron F: Boc and Gas1 each form distinct Shh receptor complexes with Ptch1 and are required for Shhmediated cell proliferation. Developmental cell 2011, 20(6):788-801.

18. Heller A, Price S, Won L: Glial-derived neurotrophic factor (GDNF) induced morphological differentiation of an immortalized monoclonal hybrid dopaminergic cell line of mesencephalic neuronal origin. Brain research 1996, 725(1):132-136.

19. Zervas M, Millet S, Ahn S, Joyner AL: Cell behaviours and genetic lineages of the mesencephalon and rhombomere 1. Neuron 2004, 43(3):345-357.

20. Ang SL: Foxa1 and Foxa2 transcription factors regulate differentiation of midbrain dopaminergic neurons. Advances in experimental medicine and biology 2009, 651:58-65.

21. Hayes L, Zhang Z, Albert P, Zervas M, Ahn S: Timing of Sonic hedgehog and Gli1 expression segregates midbrain dopamine neurons. The Journal of comparative neurology 2011, 519(15):30013018.

22. Hynes M, Porter JA, Chiang C, Chang D, Tessier-Lavigne M, Beachy PA, Rosenthal A: Induction of midbrain dopaminergic neurons by Sonic hedgehog. Neuron 1995, 15(1):35-44.

23. Pierani A, Brenner-Morton S, Chiang C, Jessell TM: A sonic hedgehog-independent, retinoid-activated pathway of neurogenesis in the ventral spinal cord. Cel/ 1999, 97(7):903-915.

24. Wijgerde M, McMahon JA, Rule M, McMahon AP: A direct requirement for Hedgehog signalling for normal specification of all ventral progenitor domains in the presumptive mammalian spinal cord. Genes \& development 2002, 16(22):2849-2864. 
25. Blaess S, Corrales JD, Joyner AL: Sonic hedgehog regulates Gli activator and repressor functions with spatial and temporal precision in the mid/hindbrain region. Development 2006, 133(9):17991809.

26. Farkas LM, Dunker N, Roussa E, Unsicker K, Krieglstein K: Transforming growth factor-beta(s) are essential for the development of midbrain dopaminergic neurons in vitro and in vivo. The Journal of neuroscience : the official journal of the Society for Neuroscience 2003, 23(12):5178-5186.

27. Joksimovic $M$, Awatramani R: Wnt/beta-catenin signalling in midbrain dopaminergic neuron specification and neurogenesis. Journal of molecular cell biology 2014, 6(1):27-33.

28. Joksimovic M, Yun BA, Kittappa R, Anderegg AM, Chang WW, Taketo MM, McKay RD, Awatramani RB: Wnt antagonism of Shh facilitates midbrain floor plate neurogenesis. Nature neuroscience 2009, 12(2):125-131.

29. Mesman S, von Oerthel L, Smidt MP: Mesodiencephalic dopaminergic neuronal differentiation does not involve GLI2A-mediated SHH-signalling and is under the direct influence of canonical WNT signalling. PloS one 2014, 9(5):e97926.

30. Tang M, Villaescusa JC, Luo SX, Guitarte C, Lei S, Miyamoto Y, Taketo MM, Arenas E, Huang EJ: Interactions of Wnt/beta-catenin signalling and sonic hedgehog regulate the neurogenesis of ventral midbrain dopamine neurons. The Journal of neuroscience : the official journal of the Society for Neuroscience 2010, 30(27):9280-9291.

31. Feuerstein M, Chleilat E, Khakipoor S, Michailidis K, Ophoven C, Roussa E: Expression patterns of key Sonic Hedgehog signalling pathway components in the developing and adult mouse midbrain and in the MN9D cell line. Cell and tissue research 2017, 370(2):211-225 \%@ 0302-0766X.

32. Cole F, Krauss RS: Microform holoprosencephaly in mice that lack the Ig superfamily member Cdon. Current biology : $C B$ 2003, 13(5):411-415.

33. Estudillo E, Zavala P, Perez-Sanchez G, Ayala-Sarmiento AE, Segovia J: Gas1 is present in germinal niches of developing dentate gyrus and cortex. Cell Tissue Res 2016, 364(2):369-384.

34. Ernsberger U, Patzke H, Tissier-Seta JP, Reh T, Goridis C, Rohrer H: The expression of tyrosine hydroxylase and the transcription factors $\mathrm{cPhox}-2$ and Cash-1: evidence for distinct inductive steps in the differentiation of chick sympathetic precursor cells. Mechanisms of development 1995, 52(1):125-136.

35. Choi HK, Won LA, Kontur PJ, Hammond DN, Fox AP, Wainer BH, Hoffmann PC, Heller A: Immortalization of embryonic mesencephalic dopaminergic neurons by somatic cell fusion. Brain research 1991, 552(1):67-76.

\section{Figures}




\section{Rostral}

\section{Caudal}

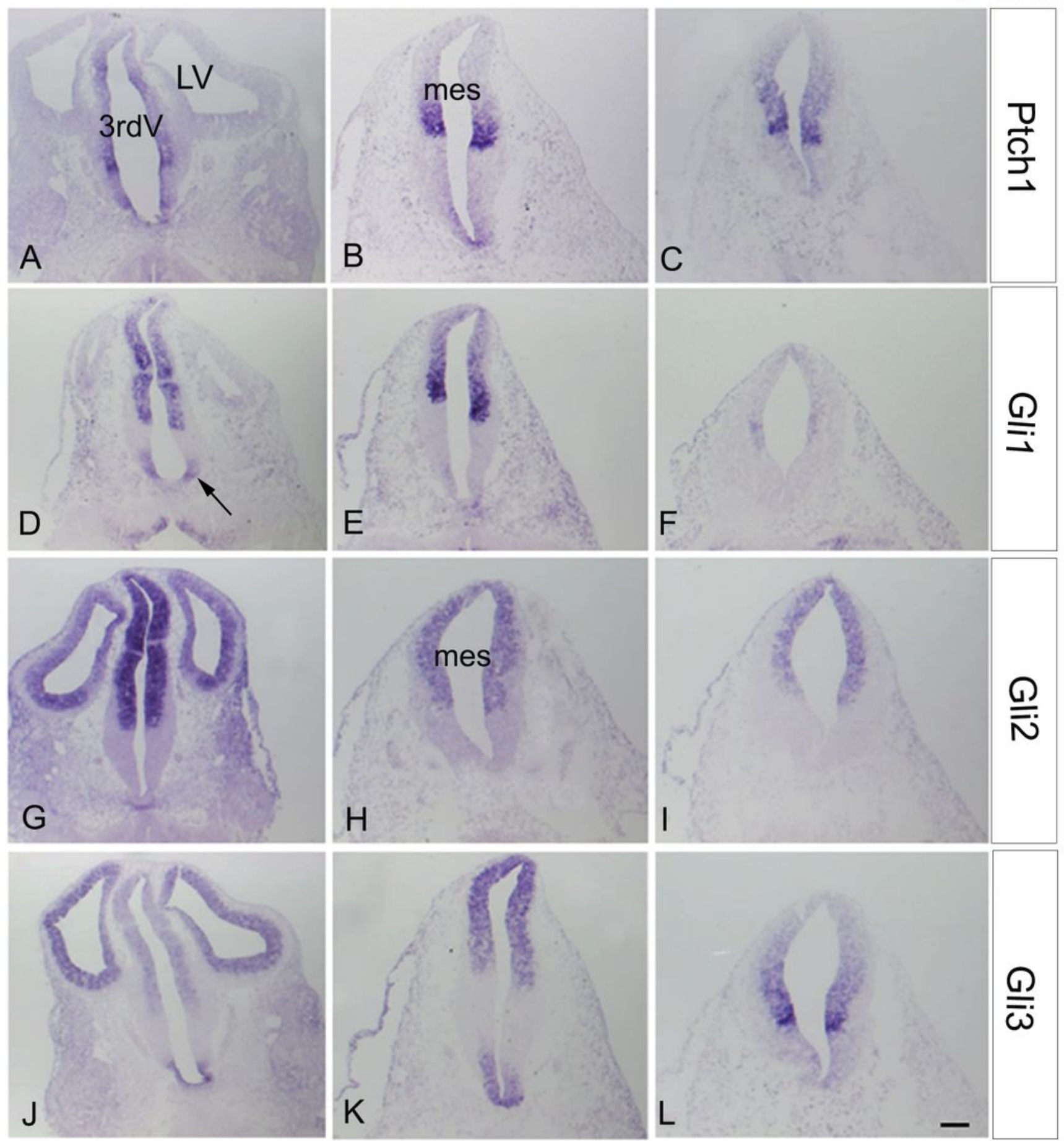

\section{Figure 1}

Expression of SHH signaling molecules in mice midbrains at embryonic day 12 (E12). In situ hybridization with Ptch1 (A-C), Gli1 (D-F), Gli2 (G-I) and Gli3 (J-L) probes on coronal frozen sections. Ptch1 and Gli1 were diffusely expressed at both the dorsoventral axis and along the rostrocaudal axis. They became progressively confined to the mediolateral domain of the mesencephalon (mes). Gli2 and 
Gli3 expression expanded also dorsally. 3rdV: third ventricle; LV: lateral ventricle. Arrow in D points to ventrolateral domain of the midbrain. Scale bar: $200 \mu \mathrm{m}$.

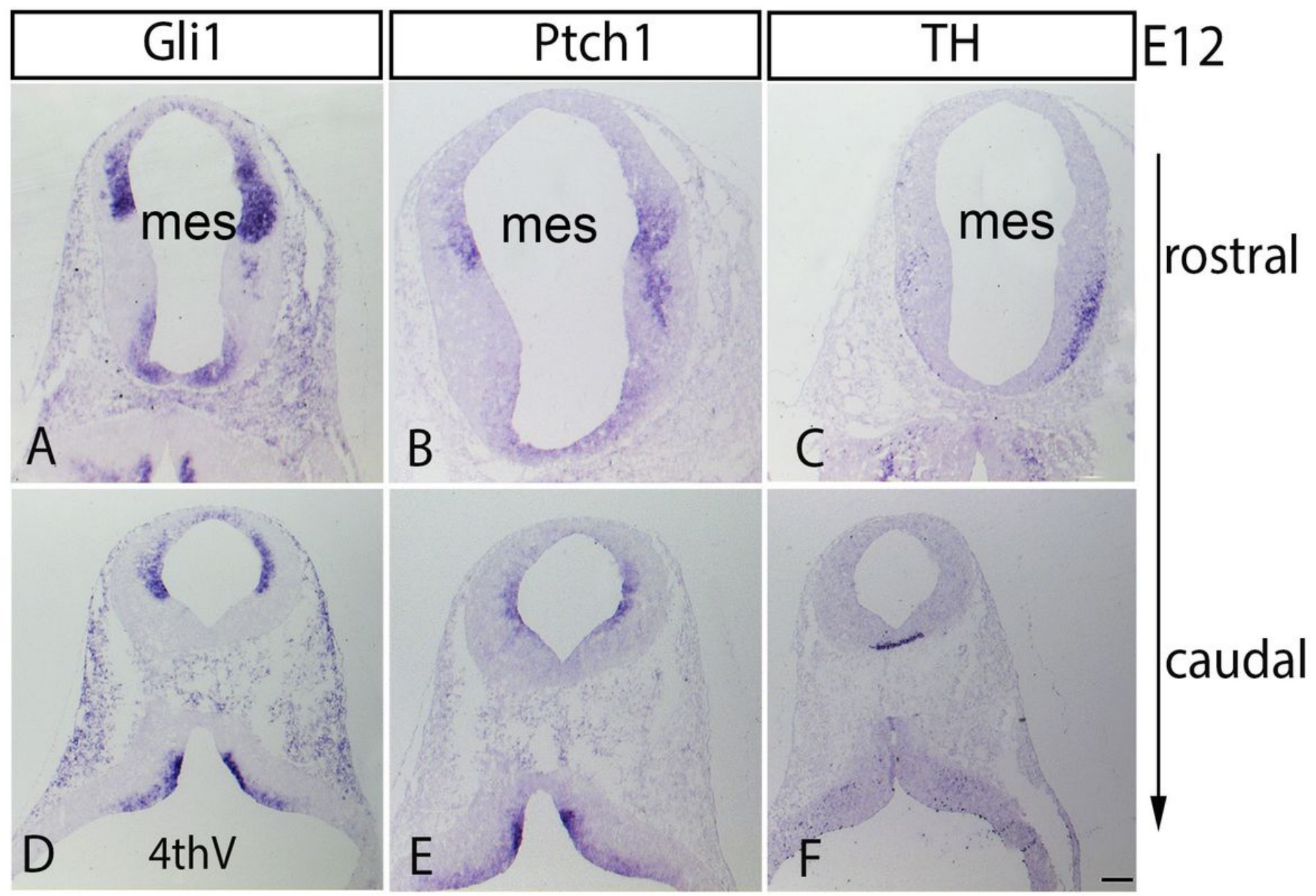

Figure 2

Expression domains of Ptch1 and Gli1 do not colocalize with TH at E12 mouse midbrain. Gli1in situ hybridization (A, D), Patch1 $(B, E)$ and TH $(C, F)$ probes on E12 coronal fixed cryosections. Mes: mesencephalon; 4th V: 4 th ventricle. Scale bar: $200 \mu \mathrm{m}$. 


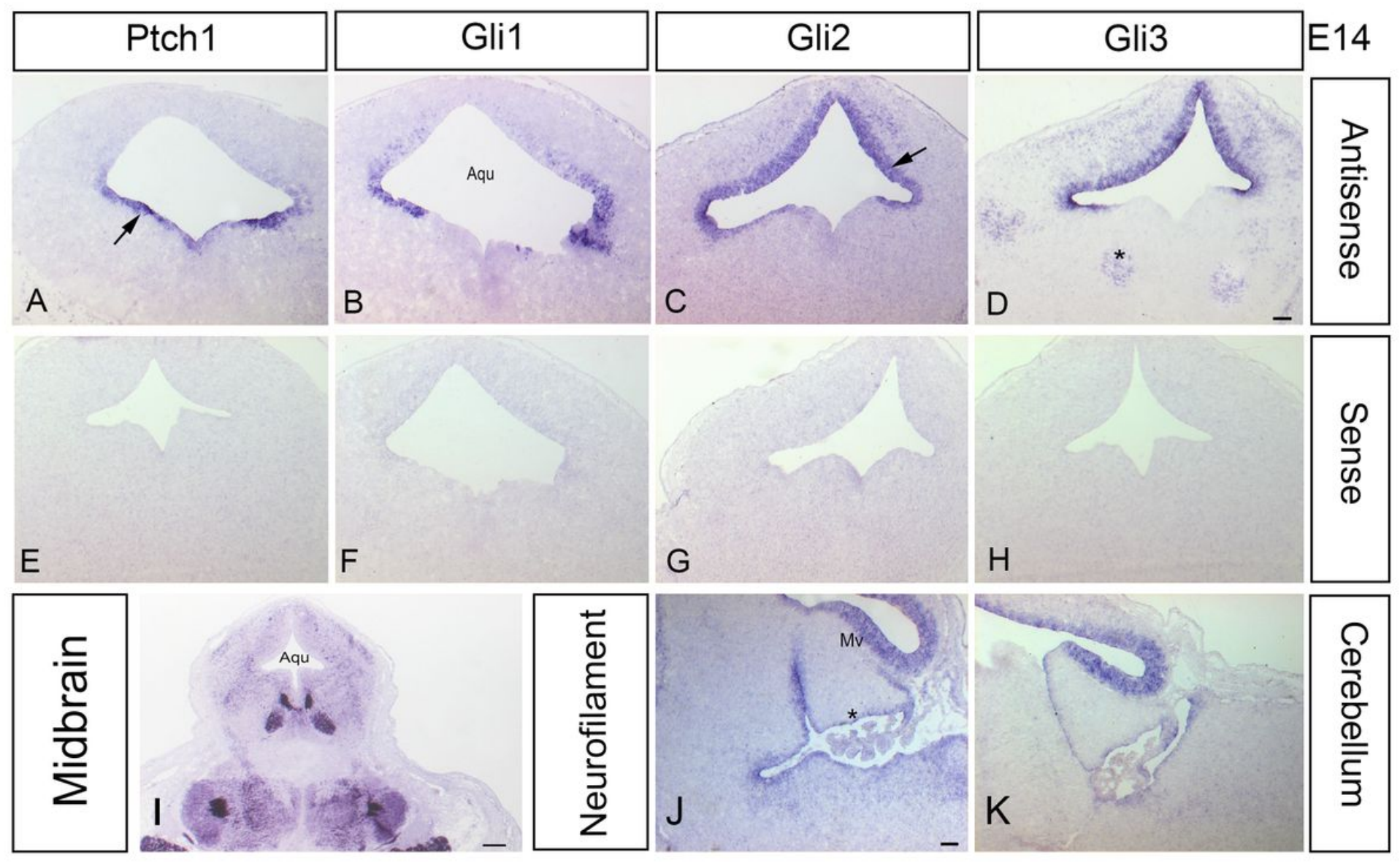

\section{Figure 3}

Expression of Ptch1and Gli family in the embryonic midbrain at E14. RNA in situ hybridization with antisense (A-D) and sense (E-H) probes on coronal midbrain cryosections. Ptch1 expression (A) at the ventrolateral midbrain (arrow),Gli2 (C; arrow) and Gli 3 (D) expression are expanded also dorsally. Gli1 expression (B) is confined to lateral midbrain domain. Gli2 and Gli3 expression were the highest among the other mRNA probes.Sense probes revealed no labeling. Expression of Neurofilament (I) on coronal midbrain cryosections, showingGli2 $(\mathrm{J})$ and Gli3 $(\mathrm{K})$ expression on sagittal developing cerebellum, the positive controls. Asterisk in $\mathrm{D}$ points to the red nucleus, in $\mathrm{J}$ to $\mathrm{Gli} 2$ expression in the cerebellum. $\mathrm{Mv}$ : mesencephalic vesicle, Aqu: aqueduct. Scale bar: $100 \mu \mathrm{m}$. 


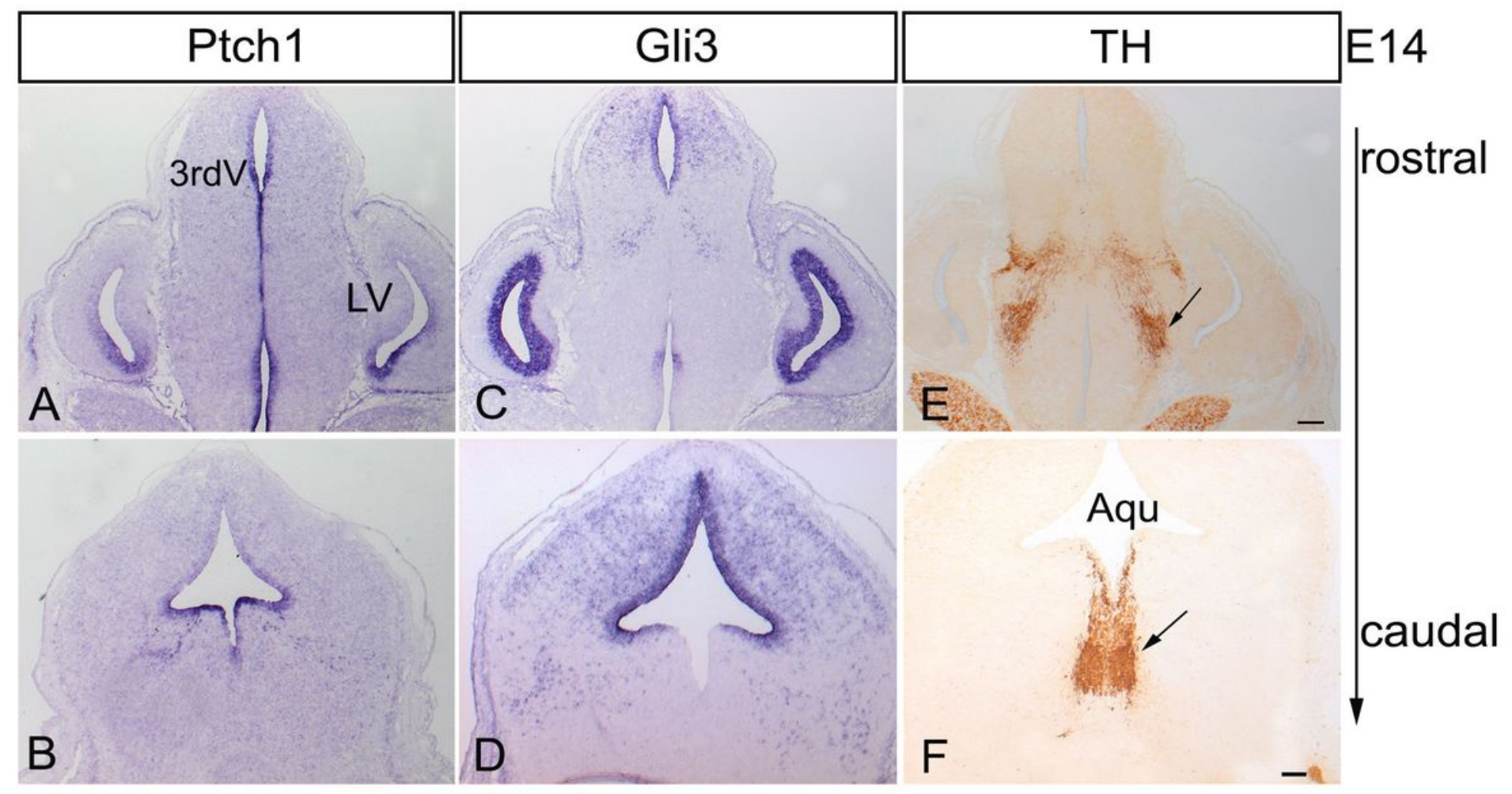

\section{Figure 4}

Lack of Ptch1 and Gli3 expression in the ventral mesencephalon at E14. In situ hybridization on coronal midbrain cryosections revealed Ptch1 (A, B), Gli3 (C, D) expression ventrally and dorsolaterally in the midbrain, respectively. $(E, F)$ Immunoperoxidase light microscopy on consecutive cryosections for tyrosine hydroxylase $(\mathrm{TH})$. Arrows indicate midbrain $\mathrm{TH}$ immunopositive dopaminergic neurons. Scale bars: in A, C, E: $200 \mu \mathrm{m}$, in B, D, F: $100 \mu \mathrm{m}$. 


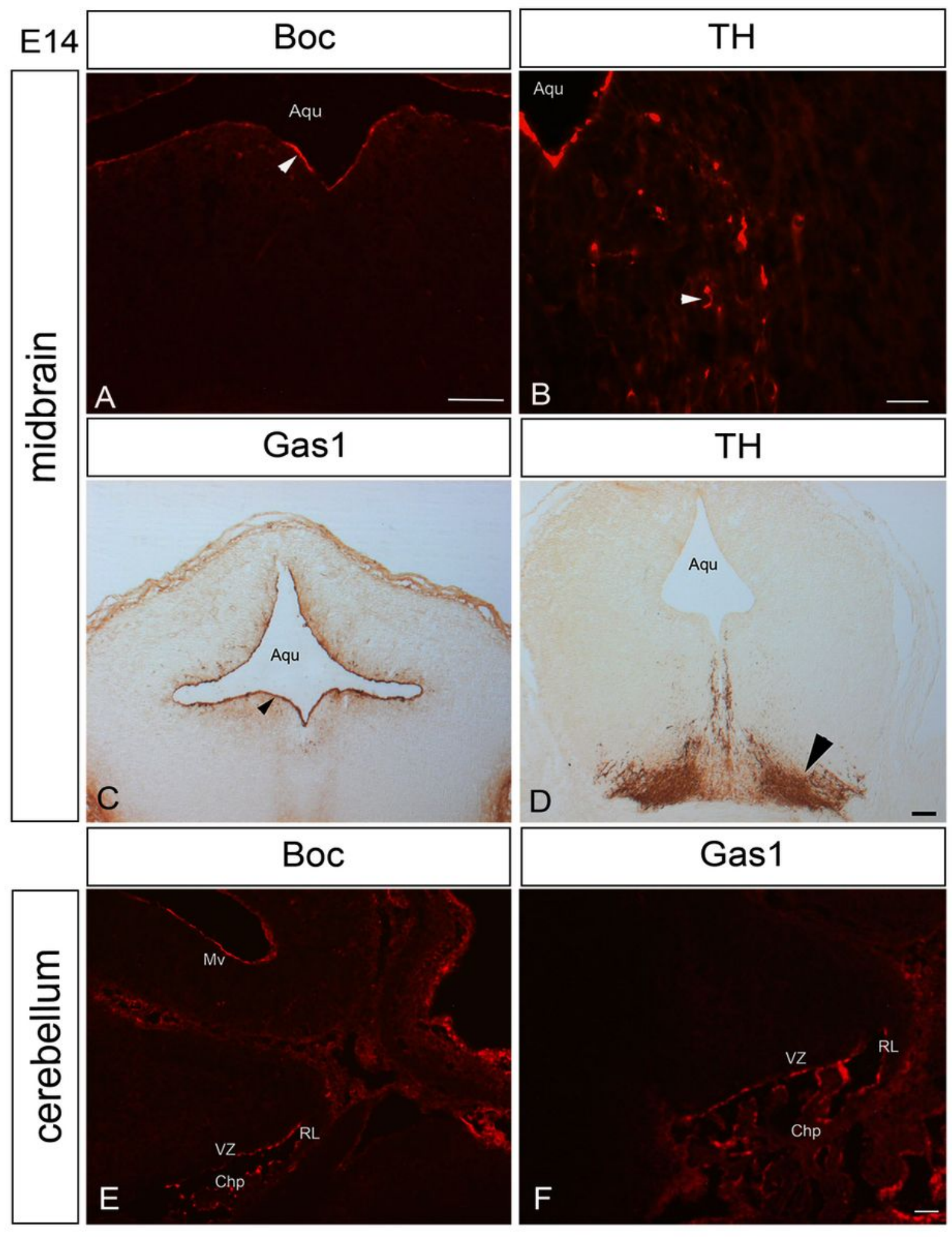

Figure 5

Boc and Gas 1 absence in mice midbrain at E14. Immunoreactivity for Boc (A) and TH (B) on coronal frozen sections showed absence of Boc immunoreactivity in TH positive neurons. (C-D) Distribution of Gas1 and TH in E14 mouse midbrain by DAB-staining light microscopy. Arrowheads point to the proliferative zone in $A$ and $C$ and to midbrain dopaminergic neurons in $B$ and $D$. (E-F) Immunofluorescence for Gas1 and Boc on sagittal cryosections of the embryonic cerebellum. Aqu: 
aqueduct, RL: rhombic lip, VZ: ventricular zone, Chp: choroid plexus, Mv: mesencephalic vesicle, Mg: meninges. Scale bars: in A, C, D: $100 \mu \mathrm{m}$, in B. $20 \mu \mathrm{m}$ and in E, F: $50 \mu \mathrm{m}$.

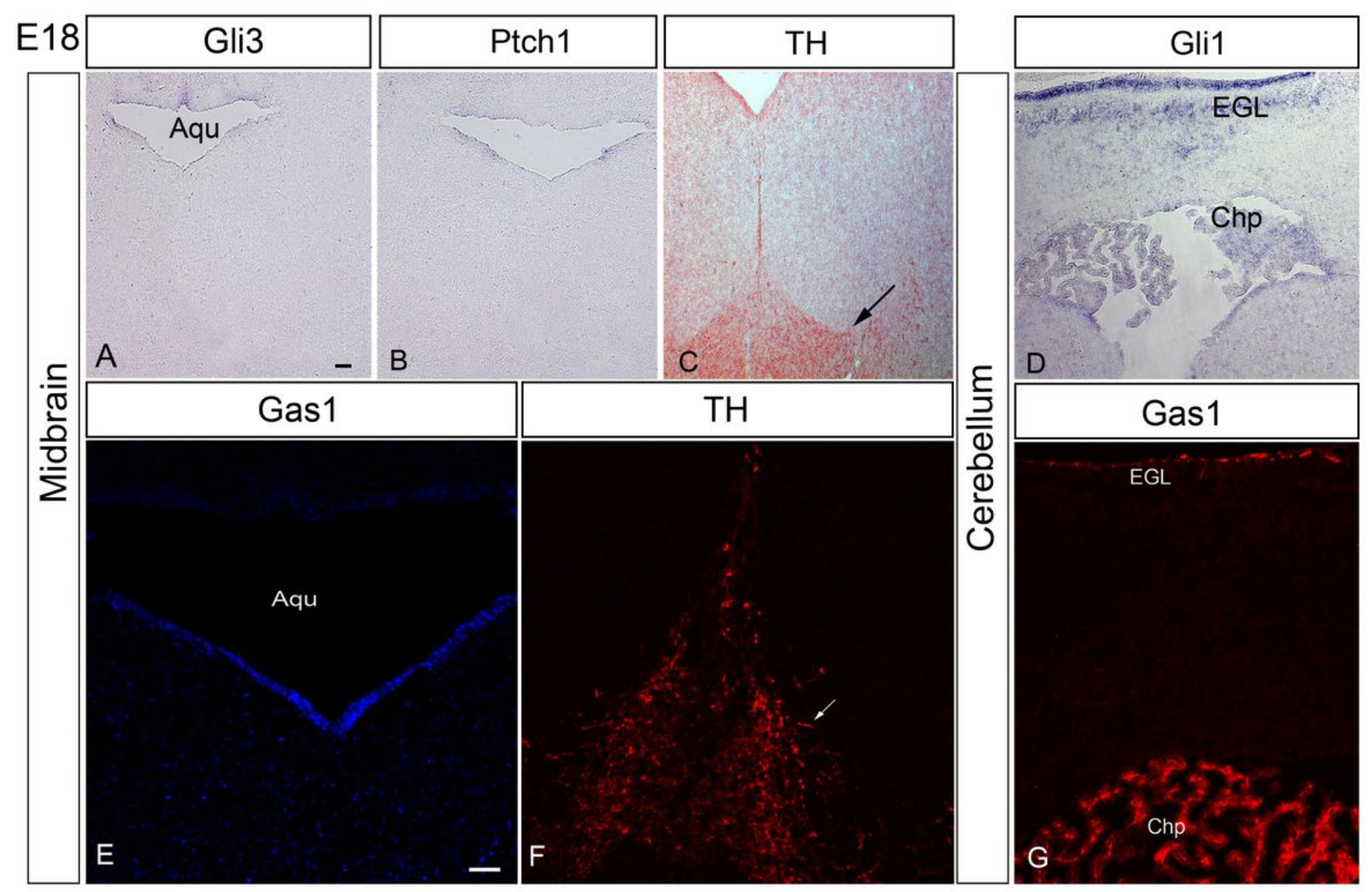

\section{Figure 6}

SHH downstream signalling molecules in embryonic mice midbrain and cerebellum at E18. In situ hybridization on coronal cryosections using specific probes against Gli3 (A), Ptch1 (B) and Gli1 (D). Consecutive midbrain sections were immuno-labelled against TH (C, F). Arrows indicate mesencephalic dopaminergic neurons. Gas1/DAPI expression in the late embryonic midbrain (E) is not detectable. D, G: Gli1 and Gas-1 expression in the cerebellum. EGL: external germinal layer, Chp: choroid plexus, Aqu: aqueduct. Scale bars: in A-D: $100 \mu \mathrm{m}$, in E-G: $50 \mu \mathrm{m}$. 

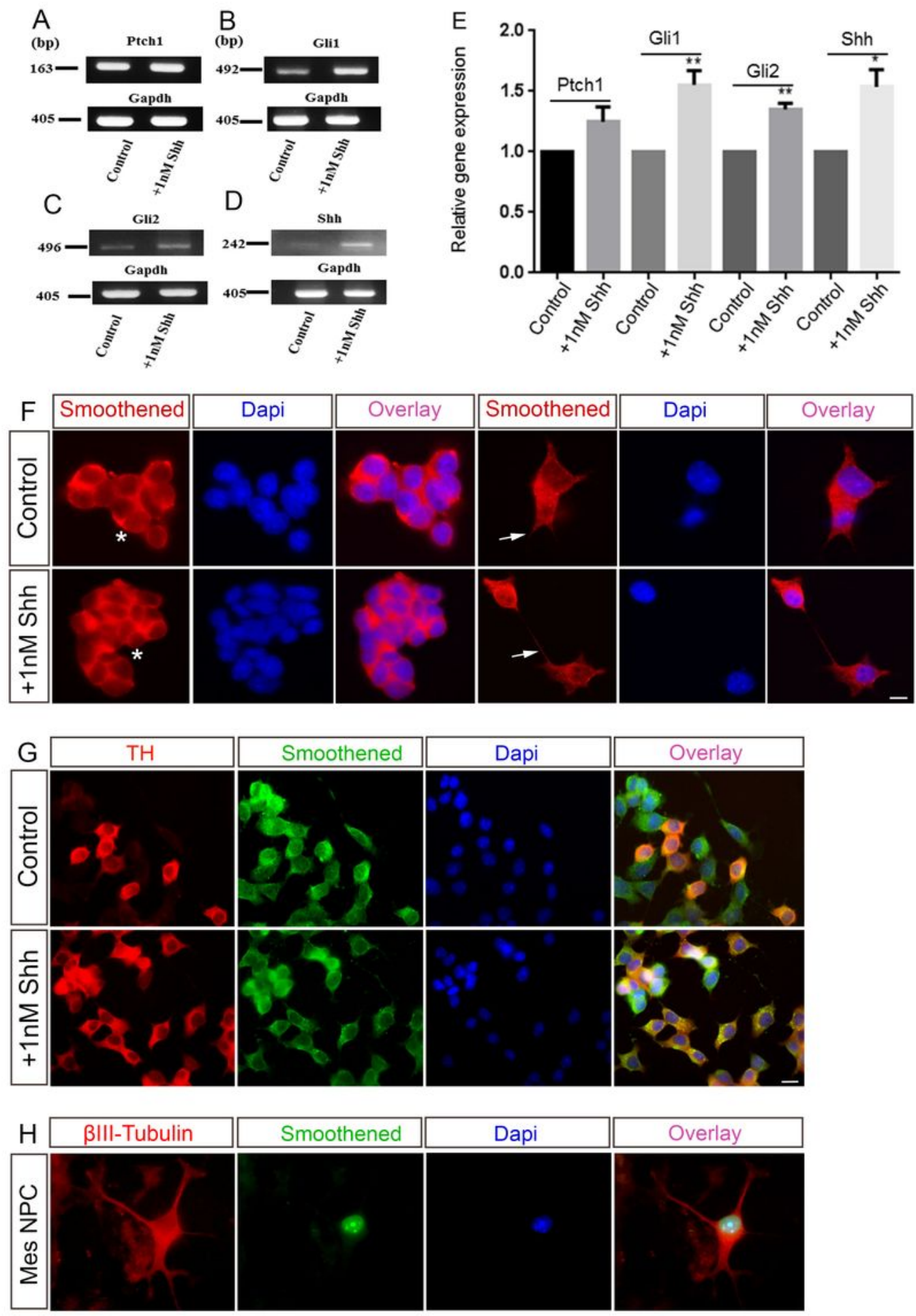

\section{Figure 7}

Effects of SHH treatment on the regulation of SHH signalling molecules in MN9D cells. (A-D) Treatment of cultured MN9D cells with $1 \mathrm{nM} \mathrm{SHH}$ for 48h, followed by RT-PCR analysis and (E) Quantification of the data. The expression of the examined genes transcripts was normalized to GAPDH. ${ }^{*} p<0.05$, and ${ }^{\star *} p<0.01$ indicate significant statistic differences relative to control, as assessed by an unpaired Student's t-test $(n=3)$. Data are given as fold changes compared to control. (F) Treated MN9D cells with 1nM SHH 
for 48h, followed by immuno-labelling for Smoothened (red). Asterisks indicate undifferentiated MN9D cells, whereas, arrows point to MN9D cells with neuronal phenotype. (G) SHH-treated MN9D cells and controls were positive for Smoothened (green), independent of both their differentiation conditions and treatment with exogenous Shh, because not all Smoothened expressing cells are TH positive (red). $(H)$ Primary culture of mouse mesencephalic neural progenitor cells (Mes NPC) at E14. Double Immunofluorescent staining revealed that differentiated neuronal cells ( $\beta$ III-tubulin immunoreactive cells) were not expressing Smoothened (green). Scale bar: $10 \mu \mathrm{m}$.

\section{Supplementary Files}

This is a list of supplementary files associated with this preprint. Click to download.

- NC3RsARRIVEGuidelinesChecklistfillable.pdf

- BMCoriginaldata1.pdf 